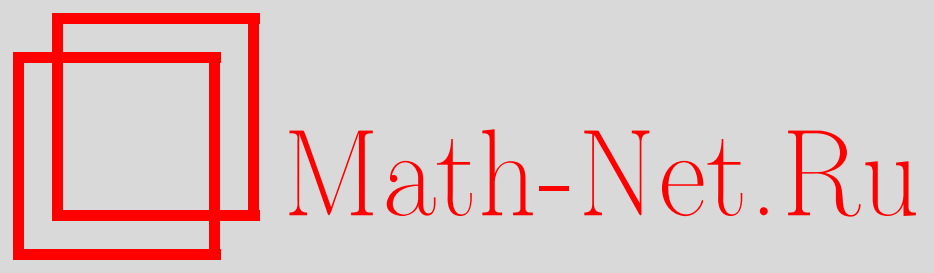

K. А. Попков, Проверяющие и диагностические тесты для функциональных элементов, Дискрет. матем., 2014, том 26, выпуск 2, 83-99

DOI: https://doi.org/10.4213/dm1282

Использование Общероссийского математического портала Math-Net.Ru подразумевает, что вы прочитали и согласны с пользовательским соглашением http://www . mathnet.ru/rus/agreement

Параметры загрузки:

IP : 54.237 .206 .68

26 апреля 2023 г., 12:34:50

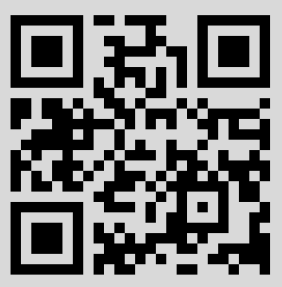




\title{
Проверяющие и диагностические тесты для функциональных элементов
}

\author{
() 2014 г. K. А. Попков*
}

\begin{abstract}
Рассматриваются задачи проверки исправности и диагностики состояний $N$ функциональных элементов, реализующих в исправном состоянии заданную булеву функцию $f\left(x_{1}, \ldots, x_{n}\right)$, путём составления из них схем с одним выходом и наблюдения выдаваемых этими схемами значений на любых входных наборах значений переменных. Допускаются произвольные константные неисправности на выходах функциональных элементов; при этом предполагается, что не более $k$ элементов неисправны, где $k$ - заданное натуральное число, не превосходящее $N$. Требуется минимизировать число схем, необходимых для проверки исправности и определения состояний всех функциональных элементов. Доказано, что, если $f \notin\left\{x_{1} \& \ldots \& x_{n}, x_{1} \vee \ldots \vee x_{n}, \overline{x_{1}}\right\}$ и выполнено некоторое условие на $k$ и $N$, то для проверки исправности всех элементов достаточно $2 k+1$ схем. Если при этом функция $f$ нелинейна, то для определения состояний всех элементов также достаточно $2 k+1$ схем.
\end{abstract}

Ключевые слова: функциональный элемент, неисправность, схема, проверяющий тест, диагностический тест.

\section{Введение}

В статье рассматриваются задачи проверки исправности и распознавания состояний функциональных элементов с использованием экспериментов, заключающихся в составлении произвольных схем из заданных функциональных элементов с последующим "прозваниванием" этих схем, т. е. нахождением булевых функций, реализуемых составляемыми схемами. Суть общепринятой математической модели схемы из функциональных элементов и тех элементов, из которых строятся эти схемы, с исчерпывающей полнотой и ясностью представлена в [1]; именно такая математическая модель является объектом исследования и рассматривается ниже.

Представим, что имеются $N$ функциональных элементов $E_{1}, \ldots, E_{N}(N \geqslant 1)$. Каждый элемент, рассматриваемый как простейшая схема из функциональных элементов, имеет $n \geqslant 1$ входов $v_{1}, \ldots, v_{n}$ и один выход и в исправном состоянии реализует на выходе заданную булеву функцию $f\left(x_{1}, \ldots, x_{n}\right)$, где $x_{1}, \ldots, x_{n}$ - переменные, подаваемые на его входы, соответственно, $v_{1}, \ldots, v_{n}$ (считаем, что функция $f\left(x_{1}, \ldots, x_{n}\right)$ существенно зависит от всех своих переменных и, как следствие, отлична от константы). В неисправном состоянии каждый элемент реализует одну из констант 0 или 1 . Неисправность элемента $E_{i}$, при которой он реализует константу

*Место работы: МГУ им. М.В.Ломоносова, e-mail: kirill-formulist@mail.ru 
0 (или 1), будем называть неисправностью $E_{i}$ типа 0 (соответственно, 1). Предполагается, что среди данных $N$ функциональных элементов не более $k$ элементов могут быть неисправны, где $k$ - заданное натуральное число, $k \leqslant N$. Можно составлять любые схемы с одним выходом из данных функциональных элементов и наблюдать выдаваемые схемами значения на любых наборах значений переменных.

Задача заключается в том, чтобы протестировать функциональные элементы, то есть для каждого из них определить, исправен данный элемент или неисправен (задача проверки), и, в дополнение к этому, определить тип неисправности каждого неисправного элемента (задача диагностики), используя при тестировании по возможности меньшее число схем.

\section{1. Основные определения и вспомогательные утвер- ждения}

Будем называть неисправностъю системы элементов любое множество неисправностей функциональных элементов при условии, что число этих неисправностей не больше $k$. (В частности, случай, когда все элементы исправны, является одним из видов неисправности системы элементов.)

Здесь и далее будем предполагать, что для любого $i$ от 1 до $N$ элемент $E_{i}$ имеет номер $i$. Тогда неисправность любого элемента можно представить в виде упорядоченной пары $\{i, \delta\}$, где $i$ - номер этого элемента, $\delta$ - булева константа, которую он реализует. Соответственно, любую неисправность системы элементов можно представить в виде множества $\left\{\left\{i_{1}, \delta_{1}\right\}, \ldots,\left\{i_{s}, \delta_{s}\right\}\right\}$, где $s$ - число неисправных элементов, $i_{1}, \ldots, i_{s}-$ номера неисправных элементов, $\delta_{j}-$ булева константа, которую реализует элемент $E_{i_{j}}$.

Диагностическим тестом назовём такой набор схем $S_{1}, \ldots, S_{l}$, составленных из заданных функциональных элементов, что для любых двух различных неисправностей системы элементов наборы функций, реализуемых схемами, не совпадают (т. е. существует такая схема $S_{j}$, что реализуемая этой схемой функция при первой неисправности не совпадает с реализуемой этой же схемой функцией при второй неисправности). Число $l$ назовём длиной этого теста. (Здесь используется терминология, общепринятая для диагностики управляющих систем: см., например, [2].)

Проверяющим тестом назовём такой набор схем $S_{1}, \ldots, S_{l}$, составленных из заданных функциональных элементов, что для любых двух неисправностей системы элементов, при которых множества неисправных элементов различны, наборы функций, реализуемых схемами, не совпадают. Число $l$ назовём длиной этого теста.

Содержательный смысл этих определений состоит в следующем: диагностический (проверяющий) тест - это такой набор схем $S_{1}, \ldots, S_{l}$, составленных из заданных $N$ функциональных элементов, что по набору функций, реализуемых этими схемами, можно однозначно определить состояние (соответственно, исправность или неисправность) каждого из $N$ элементов. При этом проверяющий тест не обязан определять тип неисправности (0 или 1) каждого неисправного элемента. 
Введём функции $L_{c}(f, N, k)$ и $L_{d}(f, N, k)$, равные длинам самого короткого, соответственно, проверяющего и диагностического тестов для $N$ функциональных элементов, среди которых не более чем $k$ неисправных (в исправном состоянии каждый элемент реализует функцию $f$ ).

Отметим, что для любых $f, N$ и $k$ выполняется соотношение

$$
L_{d}(f, N, k) \geqslant L_{c}(f, N, k),
$$

поскольку любой диагностический тест, очевидно, является проверяющим.

В качестве тривиального диагностического (и проверяющего) теста длины $N$, очевидно, всегда можно взять множество из $N$ схем, каждая из которых представляет собой один из заданных функциональных элементов. Отсюда $L_{c}(f, N, k) \leqslant N$ и $L_{d}(f, N, k) \leqslant N$ для любых $f, N$ и $k$. Легко получить также следующие нижние оценки: $L_{c}(f, N, k) \geqslant k, L_{d}(f, N, k) \geqslant k$. Действительно, если бы существовал проверяющий тест длины $l \leqslant k-1$ (состоящий, для определённости, из схем $S_{1}, \ldots, S_{l}$ ), то в силу того, что общее число функциональных элементов равно $N \geqslant k>l$, нашёлся бы такой элемент $E$ из числа $E_{1}, \ldots, E_{N}$, который не является выходным элементом ни в одной из схем $S_{1}, \ldots, S_{l}$. Тогда, скажем, при неисправности типа 0 всех выходных элементов схем $S_{1}, \ldots, S_{l}$ (а число таких элементов не превосходит $l$ ) каждая из этих схем реализовывала бы константу 0 и нельзя было бы ничего сказать относительно исправности элемента $E$, что противоречит определению проверяющего теста. Таким образом, $L_{c}(f, N, k) \geqslant k$; отсюда $L_{d}(f, N, k) \geqslant k$ в силу (1).

Итак, для любых $f, N$ и $k$ выполнены следующие тривиальные оценки:

$$
\begin{aligned}
& k \leqslant L_{c}(f, N, k) \leqslant N, \\
& k \leqslant L_{d}(f, N, k) \leqslant N .
\end{aligned}
$$

Отметим, что если на входы схем, составленных из заданных функциональных элементов, разрешить подавать наряду с переменными константы 0 и 1 , то значения функций $L_{c}(f, N, k)$ и $L_{d}(f, N, k)$ от этого не изменятся. В самом деле, поскольку класс допустимых схем будет расширен, то эти значения не увеличатся. С другой стороны, пусть некоторый набор схем $S_{1}, \ldots, S_{l}$ из этого расширенного класса является диагностическим (проверяющим) тестом, и пусть $y_{0}$ и $y_{1}$ - переменные, отличные от всех переменных всех схем $S_{1}, \ldots, S_{l}$ и различные между собой. Подадим на все те входы схем $S_{1}, \ldots, S_{l}$, на которые подавались константы 0 и 1 , переменные, соответственно, $y_{0}$ и $y_{1}$. Полученный набор схем (обозначим его $\left\{S_{1}^{\prime}, \ldots, S_{l}^{\prime}\right\}$ ) будет уже допустимым в исходной постановке задачи (см. рис. 1). Но он также будет диагностическим (проверяющим) тестом. Действительно, при тестировании схем $S_{1}^{\prime}, \ldots, S_{l}^{\prime}$ можно ограничиться только теми входными наборами значений переменных, на которых $y_{0}=0$ и $y_{1}=1$. В этом случае схемы $S_{1}^{\prime}, \ldots, S_{l}^{\prime}$ будут очевидным образом функционировать в точности как схемы $S_{1}, \ldots, S_{l}$, а значит, по их выходным функциям (которые являются подфункциями функций, реализуемых схемами $S_{1}^{\prime}, \ldots, S_{l}^{\prime}$, при подстановке в них вместо переменных $y_{0}$ и $y_{1}$, соответственно, констант 0 и 1) можно будет однозначно определить состояние (соответственно, исправность или неисправность) каждого функционального элемента. Отсюда ясно, что набор $\left\{S_{1}^{\prime}, \ldots, S_{l}^{\prime}\right\}$ является диагностическим (проверяющим) тестом в исходной постановке задачи. Таким образом, для каждого диагностического (проверяющего) теста в расширенном классе схем можно построить соответствующий тест такой же 

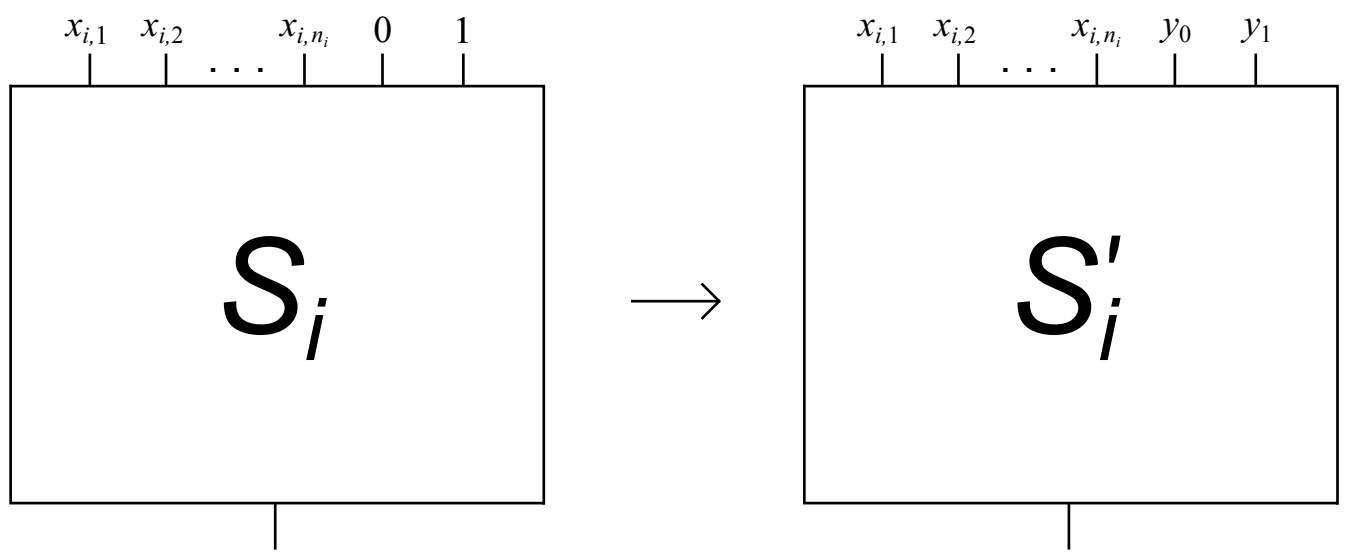

\section{( $i$ - любое число от 1 до $l$ )}

Рис. 1

длины в исходной постановке задачи, откуда следует, что после расширения класса схем значения функций $L_{c}(f, N, k)$ и $L_{d}(f, N, k)$ не уменьшатся.

Поскольку далее нас будут интересовать оценки для функций $L_{c}(f, N, k)$ и $L_{d}(f, N, k)$, будем без ограничения общности считать, что на входы схем разрешается подавать константы 0 и 1.

Пусть функциональный элемент $E$ имеет два входа и реализует в исправном состоянии функцию вида $x_{1}^{\sigma_{1}} \& x_{2}^{\sigma_{2}}$ или $x_{1}^{\sigma_{1}} \vee x_{2}^{\sigma_{2}}$, где $\sigma_{1}, \sigma_{2}$ - некоторые булевы константы. Тогда назовём его вход, отвечающий переменной $x_{i}, i=1,2$, nрямым, если $\sigma_{i}=1$, и инверсионным, если $\sigma_{i}=0$.

\section{2. Формулировка и доказательство основного резуль- тата}

Основным результатом данной работы является следующая теорема, позволяющая в ряде случаев существенно улучшить верхние оценки для функций $L_{c}(f, N, k)$ и $L_{d}(f, N, k)$ в $(2)$ и $(3)$ и приблизить их к нижним.

Теорема 1. Пусть булева функиия $f\left(x_{1}, \ldots, x_{n}\right)$ не совпадает ни с одной из функиий $x_{1} \& \ldots \& x_{n}, x_{1} \vee \ldots \vee x_{n}, \overline{x_{1}}$ и выполнено условие $8 k+\frac{5}{2} \leqslant \sqrt{N}$. Тогда:

1) $L_{c}(f, N, k) \leqslant 2 k+1$,

2) если функиия $f\left(x_{1}, \ldots, x_{n}\right)$ нелинейна, то $L_{d}(f, N, k) \leqslant 2 k+1$.

Доказательство распадается на ряд этапов; некоторые из них представлены в виде лемм.

По условию рассматриваемой задачи функция $f\left(x_{1}, \ldots, x_{n}\right)$ существенно зависит от всех своих переменных и отлична от константы; из условий теоремы следует, что $f$ отлична также от функций $x_{1}, \overline{x_{1}}$. Поэтому $n \geqslant 2$.

Лемма 1. Из функиии $f\left(x_{1}, \ldots, x_{n}\right)$ отождествлением и переименованием переменных и подстановкой вместо некоторых переменных констант 0 и 1 можно 
получить функиии $f_{D}(x, y)$ и $f_{K}(x, y)$, существенно зависящие от обеих своих переменных и такие, что $f_{D}(x, y)$ не совпадает $c x \vee y, a f_{K}(x, y)$ не совпадает $c$ $x \& y$.

\section{Доказательство. Рассмотрим два случая.}

1. Функция $f\left(x_{1}, \ldots, x_{n}\right)$ монотонна. По условию теоремы данная функция не совпадает ни с одной из функций $x_{1} \& \ldots \& x_{n}, x_{1} \vee \ldots \vee x_{n}$. Воспользуемся известным фактом о том, что из монотонной функции, существенно зависящей по крайней мере от двух переменных и не являющейся конъюнкцией, путём отождествления и переименования переменных и подстановки вместо некоторых переменных константы 1 можно получить функцию $x \vee y$ (см., например, [3], стр. 19, утверждение 4). По принципу двойственности (см., например, [3], стр. 19, утверждение 3) из этого факта следует, что из монотонной функции, существенно зависящей по крайней мере от двух переменных и не являющейся дизъюнкцией, путём отождествления и переименования переменных и подстановки вместо некоторых переменных константы 0 можно получить функцию $x \& y$. Тогда, положив $f_{K}(x, y)=x \vee y$, a $f_{D}(x, y)=x \& y$, получим утверждение леммы.

2. Функция $f\left(x_{1}, \ldots, x_{n}\right)$ не монотонна. Тогда существуют два таких соседних набора $\tilde{\sigma}_{0}=\left(\sigma_{1}, \ldots, \sigma_{i-1}, 0, \sigma_{i+1}, \ldots, \sigma_{n}\right)$ и $\tilde{\sigma}_{1}=\left(\sigma_{1}, \ldots, \sigma_{i-1}, 1, \sigma_{i+1}, \ldots, \sigma_{n}\right), i \in$ $\{1, \ldots, n\}$, что $f\left(\tilde{\sigma}_{0}\right)=1$ и $f\left(\tilde{\sigma}_{1}\right)=0$. Без ограничения общности будем считать, что $i=n$, т. е. $\tilde{\sigma}_{0}=\left(\sigma_{1}, \ldots, \sigma_{n-1}, 0\right), \tilde{\sigma}_{1}=\left(\sigma_{1}, \ldots, \sigma_{n-1}, 1\right)$. Рассмотрим следующую последовательность функций:

$$
\begin{aligned}
& f_{1}\left(x_{1}, \ldots, x_{n}\right)=f\left(x_{1}, \ldots, x_{n}\right), \\
& f_{2}\left(x_{2}, \ldots, x_{n}\right)=f\left(\sigma_{1}, x_{2}, \ldots, x_{n}\right), \\
& f_{3}\left(x_{3}, \ldots, x_{n}\right)=f\left(\sigma_{1}, \sigma_{2}, x_{3}, \ldots, x_{n}\right), \\
& \ldots \\
& f_{n}\left(x_{n}\right)=f\left(\sigma_{1}, \ldots, \sigma_{n-1}, x_{n}\right) .
\end{aligned}
$$

Для любого $i$ от 1 до $n$ выполняются равенства

$$
\begin{aligned}
& f_{i}\left(\sigma_{i}, \ldots, \sigma_{n-1}, 0\right)=f\left(\sigma_{1}, \ldots, \sigma_{n-1}, 0\right)=1, \\
& f_{i}\left(\sigma_{i}, \ldots, \sigma_{n-1}, 1\right)=f\left(\sigma_{1}, \ldots, \sigma_{n-1}, 1\right)=0 .
\end{aligned}
$$

(В случае $i=n$ эти равенства имеют вид $f_{n}(0)=f\left(\sigma_{1}, \ldots, \sigma_{n-1}, 0\right)=1, f_{n}(1)=$ $f\left(\sigma_{1}, \ldots, \sigma_{n-1}, 1\right)=1$.) Значит, каждая из функций $f_{1}\left(x_{1}, \ldots, x_{n}\right), f_{2}\left(x_{2}, \ldots, x_{n}\right), \ldots$, $f_{n}\left(x_{n}\right)$ существенно зависит от переменной $x_{n}$. Так как по условию $f_{1}\left(x_{1}, \ldots, x_{n}\right)$ существенно зависит по крайней мере от двух переменных, а $f_{n}\left(x_{n}\right)$ существенно зависит от одной переменной, то существует такое натуральное число $m$ на отрезке от 1 до $n-1$, что $f_{m}\left(x_{m}, \ldots, x_{n}\right)$ существенно зависит по крайней мере от двух переменных, а $f_{m+1}\left(x_{m+1}, \ldots, x_{n}\right)$ существенно зависит только от одной переменной (и эта переменная, очевидно, $x_{n}$ ). Из равенств

$$
\begin{aligned}
& f_{m+1}\left(\sigma_{m+1}, \ldots, \sigma_{n-1}, 0\right)=1 \\
& f_{m+1}\left(\sigma_{m+1}, \ldots, \sigma_{n-1}, 1\right)=0
\end{aligned}
$$

получаем, что $f_{m+1}\left(x_{m+1}, \ldots, x_{n}\right)=\overline{x_{n}}$. Разложим функцию $f_{m}\left(x_{m}, \ldots, x_{n}\right)$ по переменной $x_{m}$ :

$$
f_{m}\left(x_{m}, \ldots, x_{n}\right)=x_{m}^{\sigma_{m}} f_{m}\left(\sigma_{m}, x_{m+1}, \ldots, x_{n}\right) \vee x_{m}^{\overline{\sigma_{m}}} f_{m}\left(\overline{\sigma_{m}}, x_{m+1}, \ldots, x_{n}\right) .
$$


Заметим, что $f_{m}\left(\sigma_{m}, x_{m+1}, \ldots, x_{n}\right)=f_{m+1}\left(x_{m+1}, \ldots, x_{n}\right)=\overline{x_{n}}$, поэтому

$$
f_{m}\left(x_{m}, \ldots, x_{n}\right)=x_{m}^{\sigma_{m}} \overline{x_{n}} \vee x_{m}^{\overline{\sigma_{m}}} f_{m}\left(\overline{\sigma_{m}}, x_{m+1}, \ldots, x_{n}\right) .
$$

Заметим также, что функция $f_{m}\left(\overline{\sigma_{m}}, x_{m+1}, \ldots, x_{n}\right)$ не может быть тождественно равна $\overline{x_{n}}$, поскольку в этом случае равенство (4) приняло бы вид $f_{m}\left(x_{m}, \ldots, x_{n}\right)=$ $x_{m}^{\sigma_{m}} \overline{x_{n}} \vee x_{m}^{\overline{\sigma_{m}}} \overline{x_{n}}=\overline{x_{n}}$ и функция $f_{m}\left(x_{m}, \ldots, x_{n}\right)$ зависела бы существенно только от одной переменной. Поэтому существуют такие булевы константы $\delta_{m+1}, \ldots, \delta_{n-1}$, что функция $\psi\left(x_{n}\right)=f_{m}\left(\overline{\sigma_{m}}, \delta_{m+1}, \ldots, \delta_{n-1}, x_{n}\right)$ не совпадает с $\overline{x_{n}}$. Отсюда и из (4) получаем, что

$$
\begin{aligned}
f_{m}\left(x_{m}, \delta_{m+1}, \ldots, \delta_{n-1}, x_{n}\right) & =x_{m}^{\sigma_{m}} \overline{x_{n}} \vee x_{m}^{\overline{\sigma_{m}}} f_{m}\left(\overline{\sigma_{m}}, \delta_{m+1}, \ldots, \delta_{n-1}, x_{n}\right)= \\
& =x_{m}^{\sigma_{m}} \overline{x_{n}} \vee x_{m}^{\overline{\sigma_{m}}} \psi\left(x_{n}\right),
\end{aligned}
$$

где $\psi\left(x_{n}\right)$ - одна из функций $0,1, x_{n}$. Но это означает, как нетрудно заметить, что подстановкой констант $\sigma_{1}, \ldots, \sigma_{m-1}, \delta_{m+1}, \ldots, \delta_{n-1}$ вместо переменных $x_{1}, \ldots, x_{m-1}$, $x_{m+1}, \ldots, x_{n-1}$ и переименованием переменных $x_{m}$ и $x_{n}$ можно получить функцию $\varphi(x, y)$, существенно зависящую от обеих переменных и не совпадающую ни с одной из функций $x \vee y, x \& y$. Тогда, положив $f_{K}(x, y)=f_{D}(x, y)=\varphi(x, y)$, получим утверждение леммы. Лемма 1 доказана.

Если функция $f\left(x_{1}, \ldots, x_{n}\right)$ линейна, то из неё можно переименованием переменных и подстановкой вместо всех её переменных, кроме двух, константы 0 (для определённости) получить линейную функцию $f_{\oplus}(x, y)$, существенно зависящую от обеих своих переменных. Если же $f$ нелинейна, то воспользуемся известным фактом о том, что из любой нелинейной булевой функции путём переименования переменных и подстановки вместо некоторых переменных констант 0 и 1 можно получить нелинейную функцию $f_{L}(x, y)$ от двух переменных, существенно зависящую от обеих своих переменных (см., например, [4], стр. 39; в качестве нелинейной функции от двух переменных достаточно взять функцию $\left.\varphi\left(x_{1}, x_{2}\right)\right)$. Пусть $f_{D}(x, y)$ и $f_{K}(x, y)$ - функции, определяемые утверждением леммы 1 . Определим функции $f_{1}(x, y)$ и $f_{2}(x, y)$ следующим образом:

- если функция $f$ нелинейна и среди функций $f_{D}(x, y), f_{K}(x, y), f_{L}(x, y)$ есть функция $f^{\prime}(x, y)$, не совпадающая ни с одной из функций $x \vee y, x \& y, x \oplus y$ и $x \oplus y \oplus 1$, то положим $f_{1}(x, y)=f_{2}(x, y)=f^{\prime}(x, y)$ и назовём этот случай $A_{1}$;

- если функция $f$ нелинейна, каждая из функций $f_{D}(x, y), f_{K}(x, y), f_{L}(x, y)$ совпадает с одной из функций $x \vee y, x \& y, x \oplus y$ и $x \oplus y \oplus 1$ и среди функций $f_{D}(x, y), f_{K}(x, y)$ есть линейная функция $f^{\prime \prime}(x, y)$, то положим $f_{1}(x, y)=f_{L}(x, y), f_{2}(x, y)=f^{\prime \prime}(x, y)$ и назовём этот случай $A_{2}$ (очевидно, что в данном случае $f_{L}(x, y)$ - это одна из функций $x \& y, x \vee y)$;

- если функция $f$ нелинейна, каждая из функций $f_{D}(x, y), f_{K}(x, y), f_{L}(x, y)$ совпадает с одной из функций $x \vee y, x \& y, x \oplus y$ и $x \oplus y \oplus 1$ и среди функций $f_{D}(x, y), f_{K}(x, y)$ нет линейной функции, то очевидно, что $f_{D}(x, y)=x \& y, f_{K}(x, y)=x \vee y$ (других вариантов быть не может); положим тогда $f_{1}(x, y)=x \& y, f_{2}(x, y)=x \vee y$ и назовём этот случай $A_{3}$;

- если функция $f$ линейна, то положим $f_{1}(x, y)=f_{2}(x, y)=f_{\oplus}(x, y)$ и назовём этот случай $A_{4}$.

Из условия $8 k+\frac{5}{2} \leqslant \sqrt{N}$ теоремы следует, что $\lceil\sqrt{N}\rceil \geqslant 11$. Отсюда и из постулата Бертрана (см., например, [5], стр. 30) можно получить, что на интервале $(\lceil\sqrt{N}\rceil-$ 
$1 ; 2\lceil\sqrt{N}\rceil-4)$, т. е. на отрезке $[\lceil\sqrt{N}\rceil ; 2\lceil\sqrt{N}\rceil-5]$, содержится хотя бы одно простое число. Обозначим через $p$ любое простое число из этого отрезка. Тогда для $p$ выполнены следующие соотношения:

$$
\begin{gathered}
p \geqslant\lceil\sqrt{N}\rceil \geqslant \sqrt{N} \geqslant 8 k+\frac{5}{2}, \\
p(4 k+2)=\frac{p}{2}\left(8 k+\frac{5}{2}+\frac{3}{2}\right) \leqslant \frac{p}{2}\left(\sqrt{N}+\frac{3}{2}\right) \leqslant \frac{p}{2} \cdot \frac{N}{\sqrt{N}-\frac{3}{2}}= \\
=\frac{p N}{2 \sqrt{N}-3} \leqslant \frac{p N}{2\lceil\sqrt{N}\rceil-5} \leqslant \frac{p N}{p}=N,
\end{gathered}
$$

откуда

$$
\begin{gathered}
p \geqslant \sqrt{N}, \\
p \geqslant 2 k+1, \\
p(4 k+2) \leqslant N .
\end{gathered}
$$

Введём множество $B=\left\{E_{1}, \ldots, E_{N}\right\}$, состоящее из всех заданных функциональных элементов, и следующие его подмножества: $D_{i, j}(i=1, \ldots, 2 k+1, j=1, \ldots, p)$ - множества, состоящие из всех элементов, номера которых представимы в виде $p m+1+((j-1+m(i-1)) \bmod p)$, где $m$ - целое неотрицательное число; $G_{s}$ $(s=1, \ldots, 4 k+2)$ - множества, состоящие из всех элементов, номера которых представимы в виде $p(s-1)+t$, где $t-$ натуральное число от 1 до $p ; R_{i}=G_{2 i-1} \cup G_{2 i}$ $(i=1, \ldots, 2 k+1)$. Отметим, что множества $G_{s}$ попарно не пересекаются при различных $s$, а множества $R_{i}$ попарно не пересекаются при различных $i$; кроме того, $\left|G_{s}\right|=p$ для любого $s$ от 1 до $4 k+2$ в силу (7).

Пример 1. Вид множеств $D_{i, j}, G_{s}, R_{i}$ для $N=46, k=1, p=7$.

$$
\begin{array}{lll}
D_{1,1}=\{1,8,15,22,29,36,43\} & D_{2,1}=\{1,9,17,25,33,41\} & D_{3,1}=\{1,10,19,28,30,39\} \\
D_{1,2}=\{2,9,16,23,30,37,44\} & D_{2,2}=\{2,10,18,26,34,42,43\} & D_{3,2}=\{2,11,20,22,31,40\} \\
D_{1,3}=\{3,10,17,24,31,38,45\} & D_{2,3}=\{3,11,19,27,35,36,44\} & D_{3,3}=\{3,12,21,23,32,41,43\} \\
D_{1,4}=\{4,11,18,25,32,39,46\} & D_{2,4}=\{4,12,20,28,29,37,45\} & D_{3,4}=\{4,13,15,24,33,42,44\} \\
D_{1,5}=\{5,12,19,26,33,40\} & D_{2,5}=\{5,13,21,22,30,38,46\} & D_{3,5}=\{5,14,16,25,34,36,45\} \\
D_{1,6}=\{6,13,20,27,34,41\} & D_{2,6}=\{6,14,15,23,31,39\} & D_{3,6}=\{6,8,17,26,35,37,46\} \\
D_{1,7}=\{7,14,21,28,35,42\} & D_{2,7}=\{7,8,16,24,32,40\} & D_{3,7}=\{7,9,18,27,29,38\}
\end{array}
$$

$$
\begin{array}{ll}
G_{1}=\{1,2,3,4,5,6,7\} & R_{1}=\{1,2,3,4,5,6,7,8,9,10,11,12,13,14\} \\
G_{2}=\{8,9,10,11,12,13,14\} & \\
G_{3}=\{15,16,17,18,19,20,21\} & R_{2}=\{15,16,17,18,19,20,21,22,23,24,25,26,27,28\} \\
G_{4}=\{22,23,24,25,26,27,28\} & \\
G_{5}=\{29,30,31,32,33,34,35\} & R_{3}=\{29,30,31,32,33,34,35,36,37,38,39,40,41,42\} \\
G_{6}=\{36,37,38,39,40,41,42\} &
\end{array}
$$

(Для удобства, функциональный элемент с номером $n$ обозначаем просто $n$, где $n=1,2, \ldots, 46$.

Эти множества изображены на рис. 2. 


$$
\begin{aligned}
& D_{1,1} \backslash 181522293643 \\
& D_{1,2} \prec 291623303744 \\
& D_{1,3}<3101724313845 \\
& D _ { 1 , 4 } \longdiv { 4 1 1 1 8 2 5 3 2 3 9 4 6 } \\
& D _ { 1 , 5 } \longdiv { 5 1 2 1 9 2 6 3 3 4 0 } \\
& D _ { 1 , 6 } \longdiv { 6 1 3 2 0 2 7 3 4 4 1 } \\
& D _ { 1 , 7 } \longdiv { 7 4 2 1 2 8 3 5 4 2 }
\end{aligned}
$$

$$
D_{2,7} D_{2,6} D_{2,5} D_{2,4} D_{2,3} D_{2,2}
$$
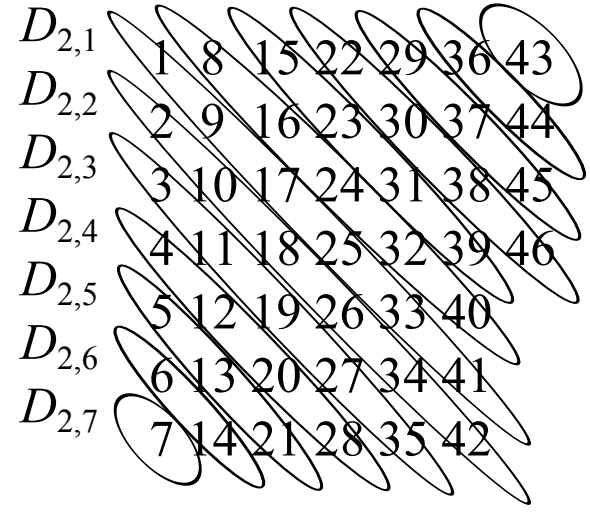

$G_{1} G_{2} G_{3} G_{4} G_{5} G_{6}$
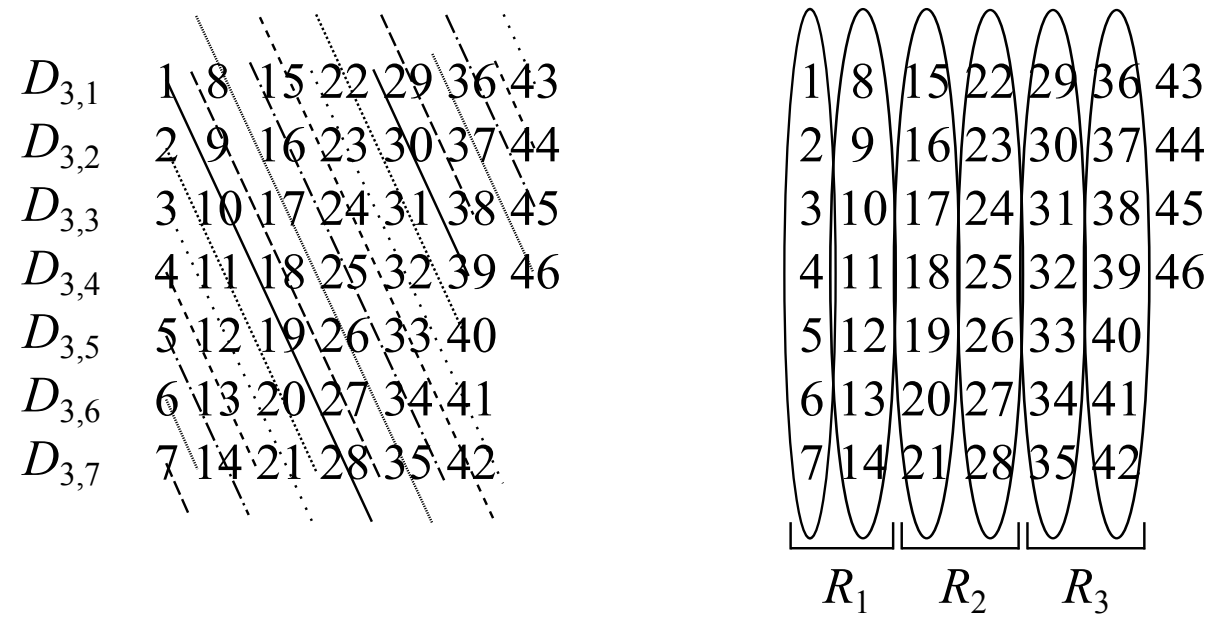

Рис. 2

Лемма 2. Справедливъ следующие утверждения.

1) Для любых таких $i \in\{1, \ldots, 2 k+1\}, j_{1}, j_{2} \in\{1, \ldots, p\}$, что $j_{1} \neq j_{2}$ выполняется соотношение $D_{i, j_{1}} \cap D_{i, j_{2}}=\varnothing$.

2) Для любого $i \in\{1, \ldots, 2 k+1\}$ выполняется соотношение $\bigcup_{j=1}^{p} D_{i, j}=B$.

3) Для любых таких $i_{1}, i_{2} \in\{1, \ldots, 2 k+1\}, j_{1}, j_{2} \in\{1, \ldots, p\}$, что $i_{1} \neq i_{2}$, множества $D_{i_{1}, j_{1}}$ и $D_{i_{2}, j_{2}}$ пересекаются не более чем по одному элементу.

4) Для любых $i \in\{1, \ldots, 2 k+1\}, j \in\{1, \ldots, p\}, s \in\{1, \ldots, 4 k+2\}$ множество $D_{i, j} \cap G_{s}$ содержит ровно один элемент.

5) Для любых $i \in\{1, \ldots, 2 k+1\}$ u $j \in\{1, \ldots, p\}$ выполняется неравенство $\left|D_{i, j}\right| \geqslant 3$. 
Справедливость утверждений 1) - 4) леммы 2 почти очевидным образом вытекает из геометрической интерпретации множеств $D_{i, j}, G_{s}$ (см. рис. 2). Фактически, данные множества представляют собой прямые или отрезки прямых в конечной аффинной плоскости порядка $p$ с $p^{2}$ точками (здесь существенно, что $p-$ простое число и выполнены соотношения (5), (6)). Определение и свойства конечных плоскостей можно найти, например, в [6], гл. 1. Для доказательства утверждения 5) достаточно заметить, что число множеств $G_{s}, s=1, \ldots, 4 k+2$, равно $4 k+2 \geqslant 3$, и воспользоваться утверждением 4).

Вернёмся к доказательству теоремы. Перейдём к построению из функциональных элементов множества $B$ схем $S_{1}, S_{2}, \ldots, S_{2 k+1}$, которые составят диагностический тест. Для любого $i \in\{1, \ldots, 2 k+1\}$ построим схему $S_{i}$ следующим образом. По утверждению 4) леммы 2 для любого $j \in\{1, \ldots, p\}$ множества $D_{i, j} \cap G_{2 i-1}$ и $D_{i, j} \cap G_{2 i}$ содержат ровно по одному элементу. Обозначим эти два элемента соответственно через $E_{i, j}^{1}$ и $E_{i, j}^{2}$. По утверждению 1$)$ леммы 2 и в силу того, что $G_{2 i-1} \cap G_{2 i}=\varnothing$, все элементы $E_{i, 1}^{1}, \ldots, E_{i, p}^{1}, E_{i, 1}^{2}, \ldots, E_{i, p}^{2}$ попарно различны. Кроме того,

$$
\begin{gathered}
\left\{E_{i, 1}^{1}\right\} \cup \ldots \cup\left\{E_{i, p}^{1}\right\}=\left(D_{i, 1} \cap G_{2 i-1}\right) \cup \ldots \cup\left(D_{i, p} \cap G_{2 i-1}\right)= \\
=\left(\bigcup_{j=1}^{p} D_{i, j}\right) \cap G_{2 i-1}=B \cap G_{2 i-1}=G_{2 i-1}, \\
\left\{E_{i, 1}^{2}\right\} \cup \ldots \cup\left\{E_{i, p}^{2}\right\}=\left(D_{i, 1} \cap G_{2 i}\right) \cup \ldots \cup\left(D_{i, p} \cap G_{2 i}\right)= \\
=\left(\bigcup_{j=1}^{p} D_{i, j}\right) \cap G_{2 i}=B \cap G_{2 i}=G_{2 i}
\end{gathered}
$$

по утверждению 2) леммы 2.

Так как функция $f\left(x_{1}, \ldots, x_{n}\right)$ существенно зависит от переменной $x_{n}$, то существуют такие булевы константы $\sigma_{1}, \ldots, \sigma_{n-1}$ (напомним, что $n \geqslant 2$ ), что функция $f\left(\sigma_{1}, \ldots, \sigma_{n-1}, x_{n}\right)$ совпадает с одной из функций $x_{n}, \overline{x_{n}}$. Пусть $j-$ произвольный индекс от 1 до $p$. Из множества $D_{i, j} \backslash\left(\left\{E_{i, j}^{1}\right\} \cup\left\{E_{i, j}^{2}\right\}\right.$ ) (оно непусто по утверждению $5)$ леммы 2) возьмём все функциональные элементы и составим из них цепь следующим образом. Сначала возьмём произвольный элемент и подадим на его входы $v_{1}, \ldots, v_{n-1}$, соответственно, константы $\sigma_{1}, \ldots, \sigma_{n-1}$, а на его вход $v_{n}$ - переменную $x_{j}$. Затем возьмём из рассматриваемого множества любой другой элемент и подадим на его входы $v_{1}, \ldots, v_{n-1}$, соответственно, константы $\sigma_{1}, \ldots, \sigma_{n-1}$, а его вход $v_{n}$ соединим с выходом предыдущего элемента. Затем возьмём из рассматриваемого множества любой элемент, отличный от первых двух, и подадим на его входы $v_{1}, \ldots, v_{n-1}$, соответственно, константы $\sigma_{1}, \ldots, \sigma_{n-1}$, а его вход $v_{n}$ соединим с выходом предыдущего элемента, и т.д. В итоге получим цепь, содержащую все элементы из $D_{i, j} \backslash\left(\left\{E_{i, j}^{1}\right\} \cup\left\{E_{i, j}^{2}\right\}\right)$. Обозначим эту цепь через $L_{i, j}$. Из её построения легко следует, что функция, реализуемая на выходе её нижнего элемента (в случае, если все элементы из $L_{i, j}$ исправны), совпадает с одной из функций $x_{j}, \overline{x_{j}}$.

Отметим, что для любых таких $j_{1}, j_{2} \in\{1, \ldots, p\}$, что $j_{1} \neq j_{2}$, множества $D_{i, j_{1}} \backslash$ $\left(\left\{E_{i, j_{1}}^{1}\right\} \cup\left\{E_{i, j_{1}}^{2}\right\}\right)$ и $D_{i, j_{2}} \backslash\left(\left\{E_{i, j_{2}}^{1}\right\} \cup\left\{E_{i, j_{2}}^{2}\right\}\right)$ не пересекаются по утверждению 1$)$ леммы 2. Таким образом, построенные нами цепи элементов $L_{i, 1}, \ldots, L_{i, p}$ попарно не пересекаются.

Далее, для любого $j \in\{1, \ldots, p\}$ рассмотрим элементы $E_{i, j}^{1}$ и $E_{i, j}^{2}$. Ни один из них не входит ни в одну из построенных ранее цепей $L_{i, 1}, \ldots, L_{i, p}$ (для цепи $L_{i, j}$ 
это верно по построению, для всех остальных цепей - по определению элементов $E_{i, j}^{1}, E_{i, j}^{2}$ и утверждению 1$)$ леммы 2). Путём отождествления некоторых входов и подачи на некоторые входы констант 0 и 1 из $E_{i, j}^{1}$ можно получить элемент $E_{i, j}^{\prime}$, имеющий два входа $v_{1}$ и $v_{2}$ и реализующий (в случае, если $E_{i, j}^{1}$ исправен) функцию $f_{1}(x, y)$, а из $E_{i, j}^{2}$ - элемент $E_{i, j}^{\prime \prime}$, имеющий два входа $v_{1}$ и $v_{2}$ и реализующий (в случае, если $E_{i, j}^{2}$ исправен) функцию $f_{2}(x, y)$, где $x, y$ - переменные, подаваемые соответственно на входы $v_{1}$ и $v_{2}$ этих элементов, что мы и сделаем для построения схемы $S_{i}$ (определение функций $f_{1}(x, y)$ и $f_{2}(x, y)$ см. в абзаце после доказательства леммы 1). У элемента $E_{i, j}^{\prime \prime}$ выделим "правый" вход (в дальнейшем, кавычки при слове "правый" будем опускать) так:

- в случае $A_{1}$ функция $f_{2}(x, y) \in\{x \& \bar{y}, \bar{x} \& y, \bar{x} \& \bar{y}, x \vee \bar{y}, \bar{x} \vee y, \bar{x} \vee \bar{y}\}$. Тогда, по определению, элемент $E_{i, j}^{\prime \prime}$ имеет хотя бы один инверсионный вход. Будем считать его правым входом любой из его инверсионных входов;

- в случаях $A_{2}, A_{3}, A_{4}$ считаем правым входом элемента $E_{i, j}^{\prime \prime}$ любой из его входов.

По построению, других случаев быть не может.

Теперь соединим вход $v_{1}$ элемента $E_{i, j}^{\prime}$ с выходом нижнего элемента цепи $L_{i, j}$, а на вход $v_{2}$ элемента $E_{i, j}^{\prime}$ подадим переменную $y_{j}$. Затем соединим правый вход элемента $E_{i, j}^{\prime \prime}$ с выходом элемента $E_{i, j}^{\prime}$; на другой вход элемента $E_{i, j}^{\prime \prime}$ (будем его считать левым) подадим:

- переменную $z$, если $j=1$;

- выход элемента $E_{i, j-1}^{\prime \prime}$, если $j>1$.

Отметим, что для любых таких $j_{1}, j_{2} \in\{1, \ldots, p\}$, что $j_{1} \neq j_{2}$, ни один из элементов $E_{i, j_{1}}^{1}, E_{i, j_{1}}^{2}$ не совпадает ни с одним из элементов $E_{i, j_{2}}^{1}, E_{i, j_{2}}^{2}$, a $\bigcup_{j=1}^{p}\left(\left\{E_{i, j}^{1}\right\} \cup\right.$ $\left.\left\{E_{i, j}^{2}\right\}\right)=G_{2 i-1} \cup G_{2 i}=R_{i}$ по построению множеств $G_{2 i-1}$ и $G_{2 i}$.

Будем говорить, что элемент $E_{i, j}^{1}$ принадлежит верхнему слою, а элемент $E_{i, j}^{2}-$ нижнему слою схемы $S_{i}$. По построению и в силу $(8),(9)$ объединение верхнего и нижнего слоёв $S_{i}$ совпадает с $G_{2 i-1} \cup G_{2 i}=R_{i}$. Выход элемента $E_{i, p}^{\prime \prime}$ будем считать выходом $S_{i}$. Все переменные $x_{1}, \ldots, x_{p}, y_{1}, \ldots, y_{p}, z$ считаем различными.

Построение схемы $S_{i}$ показано на рис. 3. Очевидно, что данная схема удовлетворяет определению схемы из функциональных элементов, на входы которой могут подаваться константы 0 и 1. Кроме того, по построению, ни один из элементов из множества $B$ не входит в неё дважды. Таким образом, схема $S_{i}$ является допустимой в нашей задаче. Заметим также, что любой элемент из $B$ входит в $S_{i}$. Действительно, для любого $j \in\{1, \ldots, p\}$ все элементы из множества $D_{i, j} \backslash\left(\left\{E_{i, j}^{1}\right\} \cup\left\{E_{i, j}^{2}\right\}\right)$ принадлежат, по построению, цепи $L_{i, j}$, а элементы $E_{i, j}^{1}$ и $E_{i, j}^{2}-$ соответственно верхнему и нижнему слоям $S_{i}$, поэтому в силу утверждения 2) леммы 2 любой элемент из множества $B$ входит в $S_{i}$.

Будем считать, что для любого $j \in\{1, \ldots, p\}$ любому элементу из множества $D_{i, j}$ в схеме $S_{i}$ соответствует переменная $x_{j}$. Тогда по утверждению 2) леммы 2 любому элементу из множества $B$ в схеме $S_{i}$ соответствует какая-то переменная из числа $x_{1}, \ldots, x_{p}$.

Так как $i$ - любое натуральное число от 1 до $2 k+1$, то построен набор схем $\left\{S_{1}, S_{2}, \ldots, S_{2 k+1}\right\}$, каждая из которых является допустимой в рассматриваемой задаче. Для доказательства утверждения 1) основной теоремы достаточно доказать, что $\left\{S_{1}, S_{2}, \ldots, S_{2 k+1}\right\}-$ проверяющий тест. 


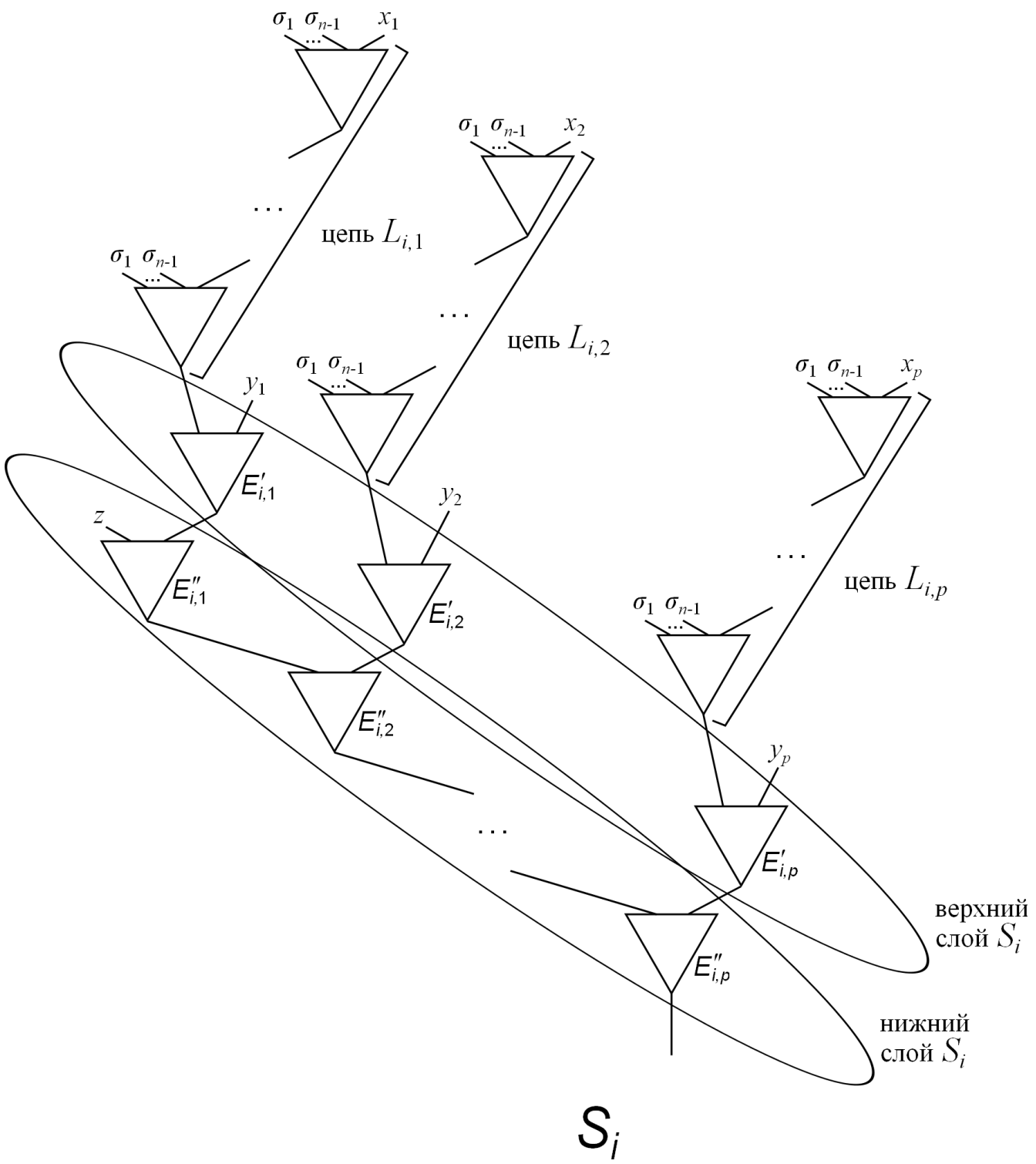

Рис 3

Отметим, что по построению в каждой из схем $S_{1}, S_{2}, \ldots, S_{2 k+1}$ каждому элементу соответствует какая-то переменная из множества $\left\{x_{1}, \ldots, x_{p}\right\}$.

Зафиксируем некоторую неисправность системы элементов. Множества $R_{1}, \ldots$, $R_{2 k+1}$ попарно не пересекаются, поэтому среди них есть хотя бы $k+1$ множеств, в каждом из которых все элементы исправны (так как неисправны не более $k$ элементов). Обозначим эти $k+1$ множеств через $R_{i_{1}}, \ldots, R_{i_{k+1}}$. Пусть $S_{i}-$ произвольная схема из числа $S_{i_{1}}, \ldots, S_{i_{k+1}}$. Рассмотрим любое $j$ от 1 до $p$. По построению, на выходе цепи $L_{i, j}$ схемы $S_{i}$ реализуется функция, зависящая от одной переменной $x_{j}$ (зависимость существенная, если все элементы в $L_{i, j}$ исправны), которая подаётся 
на один из входов элемента $E_{i, j}^{\prime}$; на другой вход этого элемента подаётся переменная $y_{j}$, а выход этого элемента подаётся на правый вход элемента $E_{i, j}^{\prime \prime}$, причём элементы $E_{i, j}^{\prime}$ и $E_{i, j}^{\prime \prime}$ двухвходовые. Так как все элементы из множества $R_{i}$ исправны, то все элементы, принадлежащие верхнему и нижнему слоям $S_{i}$, исправны, т. е. элементы $E_{i, j}^{1}$ и $E_{i, j}^{2}$ исправны. Значит, и полученные из них путём отождествления входов и подачи на некоторые входы констант 0 и 1 элементы $E_{i, j}^{\prime}$ и $E_{i, j}^{\prime \prime}$ исправны. По построению, эти элементы реализуют, соответственно, функции $f_{1}(x, y)$ и $f_{2}(x, y)$, где $x, y$ - переменные, подаваемые на их входы.

Лемма 3. Для любого $j$ от 1 до $p$ и для любой схемы $S_{i}$ из числа $S_{i_{1}}, \ldots, S_{i_{k+1}}$ на входы этой схемы вместо $x_{j}$ и $y_{j}$ можно подать такие значения, что функиця на выходе элемента $E_{i, j}^{\prime \prime}$ будет равна одной из функиий $g, \bar{g}$, где $g$ - функиия, подаваемая на левый вход этого элемента.

Доказательство. Подадим на вход схемы $S_{i}$ вместо $x_{j}$ ноль. Тогда на выходе цепи $L_{i, j}$ этой схемы будет реализована, очевидно, некоторая константа $\delta$, которая будет подаваться на вход $v_{1}$ элемента $E_{i, j}^{\prime}$; на вход $v_{2}$ этого элемента подаётся переменная $y_{j}$. Рассмотрим отдельно случаи $A_{1}, A_{2}, A_{3}$ и $A_{4}$.

Случай $A_{1}$. Тогда $f_{2}(x, y) \in\{x \& \bar{y}, \bar{x} \& y, \bar{x} \& \bar{y}, x \vee \bar{y}, \bar{x} \vee y, \bar{x} \vee \bar{y}\}$, a $f_{1}(x, y)=f_{2}(x, y)$. Рассмотрим два подслучая.

1. Функция $f_{2}(x, y)$ имеет вид $x^{\sigma_{1}} \& y^{\sigma_{2}}$, где $\sigma_{1}, \sigma_{2}$ - некоторые булевы константы. Подадим на вход схемы $S_{i}$ вместо переменной $y_{j}$ значение $\overline{\sigma_{2}}$. Тогда, в силу того, что $f_{1}(x, y)=f_{2}(x, y)$, на выходе элемента $E_{i, j}^{\prime}$ будет реализована функция $\delta^{\sigma_{1}} \& \bar{\sigma}_{2} \sigma_{2}$, т. е. тождественный ноль. Он будет подаваться на правый вход элемента $E_{i, j}^{\prime \prime}$, который, по построению, инверсионный. Это означает, что функция на выходе $E_{i, j}^{\prime \prime}$ будет равна $g^{\sigma} \& \overline{0}=g^{\sigma}$, где $g-$ функция, подаваемая на его левый вход, а $\sigma-$ булева константа, равная 1 , если его левый вход прямой, и равная 0 , если он инверсионный.

2. Функция $f_{2}(x, y)$ имеет вид $x^{\sigma_{1}} \vee y^{\sigma_{2}}$, где $\sigma_{1}, \sigma_{2}$ - некоторые булевы константы. Подадим на вход схемы $S_{i}$ вместо переменной $y_{j}$ значение $\sigma_{2}$. Тогда, в силу того, что $f_{1}(x, y)=f_{2}(x, y)$, на выходе элемента $E_{i, j}^{\prime}$ будет реализована функция $\delta^{\sigma_{1}} \vee \sigma_{2}^{\sigma_{2}}$, т. е. тождественная единица. Она будет подаваться на правый вход элемента $E_{i, j}^{\prime \prime}$, который, по построению, инверсионный. Это означает, что функция на выходе $E_{i, j}^{\prime \prime}$ будет равна $g^{\sigma} \vee \overline{1}=g^{\sigma}$, где $g$ - функция, подаваемая на его левый вход, а $\sigma-$ булева константа, равная 1 , если его левый вход прямой, и 0 , если он инверсионный.

Других подслучаев, очевидно, нет.

Случаи $A_{2}$ и $A_{4}$. Тогда $f_{2}(x, y)$ - функция вида $x \oplus y \oplus \sigma$, где $\sigma$ - некоторая булева константа, а $f_{1}(x, y)$ - одна из функций $x \& y, x \vee y, x \oplus y \oplus \sigma$. Подадим на вход схемы $S_{i}$ вместо переменной $y_{j}$ ноль. Тогда на выходе элемента $E_{i, j}^{\prime}$ будет реализована одна из функций $\delta \& 0, \delta \vee 0, \delta \oplus 0 \oplus \sigma$, т. е. некоторая булева константа $\delta^{\prime}$. Она будет подаваться на правый вход элемента $E_{i, j}^{\prime \prime}$, реализующего функцию вида $x \oplus y \oplus \sigma$. Это означает, что функция на выходе $E_{i, j}^{\prime \prime}$ будет равна $g \oplus \delta^{\prime} \oplus \sigma$, т. е. либо $g$, либо $\bar{g}$, где $g$ - функция, подаваемая на его левый вход.

Случай $A_{3}$. Тогда $f_{1}(x, y)=x \& y, f_{2}(x, y)=x \vee y$. Подадим на вход схемы $S_{i}$ вместо переменной $y_{j}$ ноль. Тогда на выходе элемента $E_{i, j}^{\prime}$ будет реализована функция $\delta \& 0$, т. е. тождественный ноль. Он будет подаваться на правый вход элемента $E_{i, j}^{\prime \prime}$, реализующего дизъюнкцию своих входов. Это означает, что функция на выходе $E_{i, j}^{\prime \prime}$ 
будет равна $g \vee 0=g$, где $g-$ функция, подаваемая на его левый вход. Все случаи разобраны, лемма 3 доказана.

Лемма 4. При любой неисправности системы элементов для любого исправного элемента $E$ из множества $B$ существует такая схема $S_{i}, i \in\{1, \ldots, 2 k+1\}$, что реализуемая этой схемой функция существенно зависит от соответствующей $E$ в этой схеме переменной.

Доказательство. Как было отмечено ранее, любой элемент из множества $B$, в том числе и элемент $E$, входит в каждую из схем $S_{1}, \ldots, S_{2 k+1}$. Из схем $S_{1}, \ldots, S_{2 k+1}$ выберем схемы $S_{i_{1}}, \ldots, S_{i_{k+1}}$ с исправными элементами в верхнем и нижнем слоях (см. абзац перед формулировкой леммы 3). По утверждениям 1) и 2) леммы 2 для любого $s \in\{1, \ldots, k+1\}$ элемент $E$ принадлежит ровно одному множеству из числа $D_{i_{s}, 1}, \ldots, D_{i_{s}, p}$ (пусть это множество $-D_{i_{s}, j_{s}}$ ). По утверждению 3 ) леммы 2 для любых таких $s_{1}, s_{2} \in\{1, \ldots, k+1\}$, что $s_{1} \neq s_{2}$, множества $D_{i_{s_{1}}, j_{s_{1}}}$ и $D_{i_{s_{2}}, j_{s_{2}}}$ пересекаются не более чем по одному элементу. Так как данные множества пересекаются

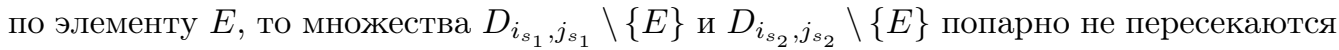
при $s_{1}, s_{2} \in\{1, \ldots, k+1\}$ и $s_{1} \neq s_{2}$. Число множеств $D_{i_{s}, j_{s}} \backslash\{E\}, s=1, \ldots, k+1$, равно $k+1$, поэтому среди них есть хотя бы одно, в котором все элементы исправны. Пусть это множество $-D_{i_{t}, j_{t}} \backslash\{E\}$. Тогда и в множестве $D_{i_{t}, j_{t}}$ все элементы исправны.

Пусть $i=i_{t}, j=j_{t}$. Рассмотрим схему $S_{i}$. По построению все элементы из множества $D_{i, j} \backslash\left(\left\{E_{i, j}^{1}\right\} \cup\left\{E_{i, j}^{2}\right\}\right)$ расположены в ней в одной цепи $L_{i, j}$, на один из входов верхнего элемента которой подаётся переменная $x_{j}$. Получаем, что все элементы в данной цепи исправны, а значит, по построению, функция, реализуемая на выходе данной цепи, существенно зависит от $x_{j}$ (обозначим эту функцию через $\varphi$; очевидно, что она совпадает с одной из функций $\left.x_{j}, \overline{x_{j}}\right)$. Затем, по построению, выход цепи $L_{i, j}$ соединяется со входом $v_{1}$ двухвходового элемента $E_{i, j}^{\prime}$, который исправен (в силу того, что он получен из элемента $E_{i, j}^{1}$, принадлежащего $R_{i}$, а в данном множестве, согласно выбору схемы $S_{i}$, все элементы исправны) и, следовательно, реализует функцию, существенно зависящую от обеих своих переменных. На вход $v_{2}$ элемента $E_{i, j}^{\prime}$ подаётся переменная $y_{j}$, значит, вместо неё можно подать такое значение, чтобы на выходе $E_{i, j}^{\prime}$ была реализована либо функция $\varphi$, либо функция $\bar{\varphi}$, т. е. либо $x_{j}$, либо $\overline{x_{j}}$, что мы и сделаем. По лемме 3 , вместо всех переменных, отличных от $x_{j}, y_{j}$, $z$, можно подать такие значения, чтобы каждый элемент из нижнего слоя, отличный от $E_{i, j}^{\prime \prime}$, реализовывал либо функцию, подаваемую на левый вход этого элемента, либо отрицание этой функции, что мы и сделаем. Так как на левый вход $E_{i, 1}^{\prime \prime}$ подаётся переменная $z$, то при $j>1$ на выходе $E_{i, 1}^{\prime \prime}$ будет реализована одна из функций $z, \bar{z}$. Эта функция будет подаваться на левый вход $E_{i, 2}^{\prime \prime}$, значит, при $j>2$ и на выходе $E_{i, 2}^{\prime \prime}$ будет реализована одна из функций $z, \bar{z}$, и т.д. В итоге, на выходе $E_{i, j-1}^{\prime \prime}$ будет реализована одна из двух функций $z, \bar{z}$. Эта функция будет подаваться на левый вход элемента $E_{i, j}^{\prime \prime}$, а правый вход данного элемента соединяется с выходом элемента $E_{i, j}^{\prime}$, на котором, как было показано, реализуется одна из двух функций $x_{j}$ и $\overline{x_{j}}$. Пусть $\psi\left(z, x_{j}\right)$ - функция, реализуемая на выходе $E_{i, j}^{\prime \prime}$, тогда $\psi\left(z, x_{j}\right)$ существенно зависит от $x_{j}$, так как элемент $E_{i, j}^{\prime \prime}$ реализует функцию, существенно зависящую от обеих своих переменных (в силу того, что данный элемент получен из элемента $E_{i, j}^{2}$, принадлежащего $R_{i}$, и, следовательно, исправен). Далее, функция $\psi\left(z, x_{j}\right)$ при $j<p$ будет подаваться на левый вход $E_{i, j+1}^{\prime \prime}$, значит, на выходе $E_{i, j+1}^{\prime \prime}$ будет реализована одна из функций $\psi\left(z, x_{j}\right), \overline{\psi\left(z, x_{j}\right)}$. Эта функция при $j+1<p$ будет 
подаваться на левый вход $E_{i, j+2}^{\prime \prime}$, значит, и на выходе $E_{i, j}^{\prime \prime}$ будет реализована одна из функций $\psi\left(z, x_{j}\right), \overline{\psi\left(z, x_{j}\right)}$, и т.д. В итоге, на выходе $E_{i, p}^{\prime \prime}$, т. е. на выходе схемы $S_{i}$, будет реализована одна из функций $\psi\left(z, x_{j}\right)$ и $\overline{\psi\left(z, x_{j}\right)}$, каждая из которых существенно зависит от переменной $x_{j}$. Это означает, что функция, реализуемая схемой $S_{i}$ при рассматриваемой неисправности системы элементов, при подстановке вместо некоторых переменных констант существенно зависит от $x_{j}$, значит, эта функция и без подстановок вместо её переменных констант существенно зависит от $x_{j}$.

Осталось заметить, что элементу $E$ в схеме $S_{i}$ соответствует именно переменная $x_{j}$. Действительно, по построению, элемент $E$ лежит в множестве $D_{i, j}$, а каждому элементу этого множества в схеме $S_{i}$ соответствует переменная $x_{j}$. Лемма 4 доказана.

Вернёмся к доказательству теоремы. Покажем, что по набору выходных функций схем $S_{1}, S_{2}, \ldots, S_{2 k+1}$ можно однозначно определить все исправные и все неисправные элементы из множества $B$. Действительно, пусть $E-$ произвольный элемент из $B$. Если ни для одной схемы из числа $S_{1}, S_{2}, \ldots, S_{2 k+1}$ функция, реализуемая этой схемой, не зависит существенно от соответствующей $E$ в этой схеме переменной, то по лемме 4 элемент $E$ неисправен. Пусть теперь хотя бы для одной схемы из числа $S_{1}, S_{2}, \ldots, S_{2 k+1}$ функция, реализуемая этой схемой, существенно зависит от соответствующей $E$ в этой схеме переменной. Обозначим эту схему через $S^{\prime}$, а переменную, соответствующую в ней $E$, через $x^{\prime}$ (по построению, $x^{\prime}$ может быть одной из переменных $\left.x_{1}, \ldots, x_{p}\right)$. Согласно выбору в схеме $S^{\prime}$ соответствующей $E$ переменной, единственная цепь из функциональных элементов, соединяющая вход $S^{\prime}$, отвечающий $x^{\prime}$, с выходом $S^{\prime}$, проходит через $E$. Отсюда, в силу того, что выходная функция $S^{\prime}$ существенно зависит от $x^{\prime}$, элемент $E$ не может реализовывать константу, т. е. исправен.

Таким образом, набор схем $S_{1}, S_{2}, \ldots, S_{2 k+1}$ позволяет определить все исправные и все неисправные элементы из множества $B$ и, следовательно, является проверяющим тестом. Его длина равна $2 k+1$, откуда следует справедливость утверждения 1 ) доказываемой теоремы.

Пусть теперь функция $f\left(x_{1}, \ldots, x_{n}\right)$ нелинейна. Докажем, что набор схем $S_{1}, S_{2}, \ldots, S_{2 k+1}$ является диагностическим тестом. Так как уже доказано, что он является проверяющим тестом, то достаточно доказать, что для любых двух различных неисправностей $H_{1}$ и $H_{2}$ системы элементов, при которых множества неисправных элементов совпадают, наборы выходных функций схем $S_{1}, S_{2}, \ldots, S_{2 k+1}$ различаются.

Так как неисправности $H_{1}$ и $H_{2}$ различны, а множества неисправных элементов при них совпадают, то существует такой элемент $E$ из $B$, что при $H_{1}$ он неисправен и выдаёт константу $\delta$, а при $H_{2}$ он неисправен и выдаёт константу $\bar{\delta}$. Множества $R_{1}, \ldots, R_{2 k+1}$ попарно не пересекаются, поэтому среди них есть хотя бы $k+1$ множеств, в каждом из которых все элементы исправны и при $H_{1}$, и при $H_{2}$ (так как неисправны не более $k$ элементов). Обозначим эти $k+1$ множеств через $R_{i_{1}}, \ldots, R_{i_{k+1}}$. По утверждениям 1$)$ и 2 ) леммы 2 для любого натурального $s \in\{1, \ldots, k+1\}$ элемент $E$ принадлежит ровно одному множеству из числа $D_{i_{s}, 1}, \ldots, D_{i_{s}, p}\left(\right.$ пусть это множество $\left.-D_{i_{s}, j_{s}}\right)$. По утверждению 3 ) леммы 2 для 
любых таких $s_{1}, s_{2} \in\{1, \ldots, k+1\}$, что $s_{1} \neq s_{2}$, множества $D_{i_{s_{1}}, j_{s_{1}}}$ и $D_{i_{s_{2}}, j_{s_{2}}}$ пересекаются не более чем по одному элементу. Так как данные множества пересекаются

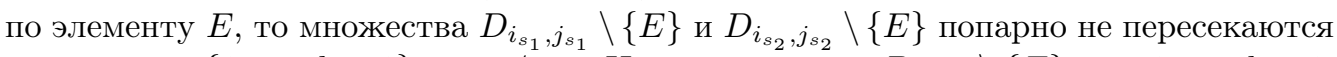
при $s_{1}, s_{2} \in\{1, \ldots, k+1\}$ и $s_{1} \neq s_{2}$. Число множеств $D_{i_{s}, j_{s}} \backslash\{E\}, s=1, \ldots, k+1$, равно $k+1$, поэтому среди них есть хотя бы одно, в котором все элементы исправны (пусть это множество $\left.-D_{i_{t}, j_{t}} \backslash\{E\}\right)$. Тогда в множестве $D_{i_{t}, j_{t}}$ единственный неисправный элемент $-E$.

Пусть $i=i_{t}, j=j_{t}$. Рассмотрим схему $S_{i}$. Элемент $E$ неисправен, поэтому он не принадлежит $R_{i}$ и, следовательно, не совпадает ни с одним из элементов $E_{i, j}^{1}, E_{i, j}^{2}$. Таким образом, элемент $E$ принадлежит множеству $D_{i, j} \backslash\left(\left\{E_{i, j}^{1}\right\} \cup\left\{E_{i, j}^{2}\right\}\right)$, т. е. цепи $L_{i, j}$, и является единственным неисправным элементом в данной цепи (так как все остальные элементы из множества $D_{i, j}$ исправны). По построению, если бы все элементы в цепи $L_{i, j}$ были исправны, то при изменении значения $x_{j}$ с 0 на 1 значение на выходе любого элемента данной цепи (в том числе $E$ ) менялось бы. Поэтому при изменении значения на выходе элемента $E$ с $\delta$ на $\bar{\delta}$ значение на выходе цепи $L_{i, j}$ меняется с 0 на 1 или с 1 на 0 . Затем, по построению, выход $L_{i, j}$ соединяется со входом $v_{1}$ двухвходового элемента $E_{i, j}^{\prime}$, на другой вход которого подаётся переменная $y_{j}$; выход $E_{i, j}^{\prime}$ соединяется с правым входом элемента $E_{i, j}^{\prime \prime}$. Заметим, что элементы $E_{i, j}^{1}$ и $E_{i, j}^{2}$ исправны, так как они оба принадлежат $R_{i}$.

Так как функция $f\left(x_{1}, \ldots, x_{n}\right)$ нелинейна, то имеет место один из случаев $A_{1}$, $A_{2}, A_{3}$. Заметим, что в каждом из данных случаев элемент $E_{i, j}^{\prime}$ реализует функцию вида либо $x^{\sigma_{1}} \& y^{\sigma_{2}}$, либо $x^{\sigma_{1}} \vee y^{\sigma_{2}}$, где $\sigma_{1}$ и $\sigma_{2}$ - некоторые булевы константы. Как мы выяснили, при изменении значения на выходе элемента $E$ с $\delta$ на $\bar{\delta}$ значение на выходе цепи $L_{i, j}$ (которое затем подаётся на вход $v_{1}$ элемента $E_{i, j}^{\prime}$ ) меняется с 0 на 1 или с 1 на 0 . Воспользуемся очевидным свойством элемента, реализующего функцию одного из видов $x^{\sigma_{1}} \& y^{\sigma_{2}}, x^{\sigma_{1}} \vee y^{\sigma_{2}}:$ при подаче одного из значений 0 или 1 на вход $v_{1}$ элемента $E_{i, j}^{\prime}$ на выходе $E_{i, j}^{\prime}$ будет реализована одна из функций $y_{j}, \overline{y_{j}}$, а при подаче другого значения на тот же вход $E_{i, j}^{\prime}$ - одна из двух функций 0,1 . Таким образом, при одной из неисправностей $H_{1}$ и $H_{2}$ (без ограничения общности, при $\left.H_{1}\right)$ на выходе $E_{i, j}^{\prime}$ реализуется одна из функций $y_{j}, \overline{y_{j}}$, а при другой $\left(H_{2}\right)-$ одна из функций 0,1 .

Применим лемму 3 и вместо всех переменных, отличных от $x_{j}, y_{j}, z$, подадим такие значения, чтобы каждый элемент из нижнего слоя, отличный от $E_{i, j}^{\prime \prime}$, реализовывал либо функцию, подаваемую на левый вход этого элемента, либо отрицание этой функции (отметим, что нам здесь неважно, какая из неисправностей $H_{1}$ и $H_{2}$ имеет место, так как схема $S_{i}$ выбирается одна и та же). Так как на левый вход $E_{i, 1}^{\prime \prime}$ подаётся переменная $z$, то при $j>1$ на выходе $E_{i, 1}^{\prime \prime}$ будет реализована одна из функций $z, \bar{z}$. Эта функция будет подаваться на левый вход $E_{i, 2}^{\prime \prime}$, значит, при $j>2$ и на выходе $E_{i, 2}^{\prime \prime}$ будет реализована одна из функций $z, \bar{z}$, и т.д. В итоге, на выходе $E_{i, j-1}^{\prime \prime}$ будет реализована одна из двух функций $z, \bar{z}$. Эта функция будет подаваться на левый вход элемента $E_{i, j}^{\prime \prime}$, а правый вход данного элемента соединяется с выходом элемента $E_{i, j}^{\prime}$, на котором, как было показано, при $H_{1}$ реализуется одна из функций $y_{j}, \overline{y_{j}}$, а при $H_{2}$ - одна из функций 0,1 . Таким образом, при $H_{2}$ на выходе $E_{i, j}^{\prime \prime}$ будет реализована функция, не зависящая существенно от $y_{j}$. Значит, при $H_{2}$ и функция на выходе всей схемы $S_{i}$ не будет зависеть существенно от $y_{j}$. При $H_{1}$ же на выходе $E_{i, j}^{\prime \prime}$ будет реализована некоторая функция $\phi\left(z, y_{j}\right)$, существенно зависящая от $y_{j}$, так как элемент $E_{i, j}^{\prime \prime}$ реализует функцию, существенно зависящую от обеих своих переменных. Далее, функция $\phi\left(z, y_{j}\right)$ при $j<p$ будет подаваться на левый вход $E_{i, j+1}^{\prime \prime}$, 
значит, на выходе $E_{i, j+1}^{\prime \prime}$ будет реализована одна из функций $\phi\left(z, y_{j}\right), \overline{\phi\left(z, y_{j}\right)}$. Эта функция при $j+1<p$ будет подаваться на левый вход $E_{i, j+2}^{\prime \prime}$, значит, и на выходе $E_{i, j}^{\prime \prime}$ будет реализована одна из функций $\phi\left(z, y_{j}\right), \overline{\phi\left(z, y_{j}\right)}$, и т.д. В итоге, на выходе $E_{i, p}^{\prime \prime}$, т. е. на выходе схемы $S_{i}$, будет реализована функция, существенно зависящая от $y_{j}$. Это означает, что функция, реализуемая схемой $S_{i}$ при неисправности $H_{1}$, при подстановке вместо некоторых переменных констант существенно зависит от $y_{j}$, откуда следует, что эта функция и без подстановок вместо её переменных констант существенно зависит от $y_{j}$. Таким образом, функции, реализуемые схемой $S_{i}$ при неисправностях $H_{1}$ и $H_{2}$, различаются (одна из них существенно зависит от $y_{j}$, а другая - нет). Следовательно, и наборы выходных функций схем $S_{1}, S_{2}, \ldots, S_{2 k+1}$ при $H_{1}$ и $H_{2}$ различаются, что и требовалось доказать.

В итоге получаем, что набор схем $S_{1}, S_{2}, \ldots, S_{2 k+1}$ является диагностичесим тестом. Его длина равна $2 k+1$, откуда следует справедливость утверждения 2) теоремы. Таким образом, теорема полностью доказана.

Замечание 1. Можно проверить, что формулировка основной теоремы может быть усилена до следующей:

Пусть булева функиия $f\left(x_{1}, \ldots, x_{n}\right)$ не совпадает ни с одной из функиий $x_{1} \vee$ $\ldots \vee x_{n}, x_{1} \& \ldots \& x_{n}, \overline{x_{1}}$, выполнено условие $\sqrt{N} \leqslant \frac{N}{4 k+2}$ и на отрезке $\left[\sqrt{N} ; \frac{N}{4 k+2}\right]$ содержится хотя бы одно натуральное число, являющееся степенъю простого числа с натуральным показателем. Тогда:

1) $L_{c}(f, N, k) \leqslant 2 k+1$,

2) если функиия $f\left(x_{1}, \ldots, x_{n}\right)$ нелинейна, mо $L_{d}(f, N, k) \leqslant 2 k+1$.

Нетрудно убедиться, что теорема в такой формулировке является усилением доказанной теоремы. Действительно, при её доказательстве от числа $p$ по существу требовалось выполнение соотношения (7) при построении множеств $G_{s}$, а также простота $p$ и выполнение соотношений (5), (6) при доказательстве утверждений 1) - 4) леммы 2. Однако при выборе в качестве $p$ любого натурального числа из отрезка $\left[\sqrt{N} ; \frac{N}{4 k+2}\right]$, являющегося степенью простого числа с натуральным показателем, соотношения (5) - (7) выполняются автоматически (с учётом неравенства $\left.\sqrt{N} \leqslant \frac{N}{4 k+2}\right)$, а конечные аффинные плоскости порядка $p$ существуют не только для простых $p$, но и для тех $p$, которые являются степенью простого числа. Таковыми, например, являются аффинные плоскости, получающиеся удалением одной прямой (вместе со всеми её точками) из проективной плоскости Галуа порядка $p$ (см. [6], $\S \S 1.1-1.8,1.10 ;$ [7], $\S \S 12.1-12.3)$. С другой стороны, теорема в новой формулировке становится верна для некоторых пар $(N ; k)$, для которых условия исходной теоремы не выполняются, например, для $(N ; k)=(42 ; 1)$ (при $p=7),(N ; k)=(224 ; 3)$ (при $p=16)$.

В заключение автор выражает глубокую благодарность своему научному руководителю профессору Н. П. Редькину за постановку задачи и внимание к работе.

\section{Список литературы}

1. Лупанов О. Б., Асимптотические оченки сложности управляющих систем, Изд-во МГУ, Москва, 1984.

2. Редькин Н. П., Надёжность и диагностика схем, Изд-во МГУ, Москва, 1992. 
3. Угольников А. Б., Классы Поста. Учебное пособие, Изд-во ЦПИ при механико-математическом факультете МГУ, Москва, 2008.

4. Яблонский С. В., Введение в дискретную математику, Наука, Москва, 1986.

5. Галочкин А. И., Нестеренко Ю. В., Шидловский А. Б., Введение в теорию чисел, Изд-во МГУ, Москва, 1984.

6. Картеси Ф., Введение в конечные геометрии, Наука, Москва, 1980.

7. Холл М., Комбинаторика, Мир, Москва, 1970.

Статья поступила 14.02.2014. 